\title{
Drug checking to improve monitoring of new psychoactive substances in Australia
}

\section{Drug checking may need to play a part in future public health interventions}

A $\mathrm{s}$ has been reported previously in the Journal, novel psychoactive stimulant drugs are now increasingly prevalent in patients presenting to hospital emergency departments. A further cluster of 11 patients showing confusing hallmarks of sympathomimetic poisoning but no identifiable substance presented to St Vincent's Hospital in Sydney over a public holiday weekend in April 2015. Also, the start to the 2015-2016 summer festival season has included multiple deaths and hospitalisations following drug use at festivals, leading to calls for novel actions to protect public health. ${ }^{2}$ Here, we take the opportunity to describe a method of harm minimisation that has been deployed in Europe and could potentially be deployed locally to tackle this problem.

\section{Monitoring new psychoactive substances}

New psychoactive substances (NPS) are emerging rapidly into the market, with more than 100 identified in the past year by European monitoring systems. ${ }^{3}$ Existing psychoactive drug monitoring systems have limited capacity to identify NPS. Their limitations are detailed as follows:

- self-reports (eg, household surveys and regular interviews with sentinel groups, as reviewed by Burns et $\mathrm{al}^{4}$ ) can identify what users think they are taking, but not necessarily what they are actually taking;

- web vendor monitoring (eg, analyses of surface web and dark web [also see Burns et $\mathrm{al}^{4}$ ]) can identify what vendors report they are selling, but not what is actually sold; and

- pharmacological analyses (eg, wastewater analyses ${ }^{5}$ ) can provide more accurate information about what is actually being consumed, but not what people believe they are consuming.

These monitoring systems have been used to identify NPS and track their use in Australia, but they are not able to characterise the congruency between what people believe they are taking and what they are actually taking. Combining all three of these methods and providing an innovative drug-checking service would strengthen surveillance of drugs being used in festival settings. Accurate and tailored information can facilitate an open dialogue between health care providers and drug users at the point of consumption. Identifying emerging trends in NPS will provide opportunities to prevent harm, and enable our services to respond more effectively to the harms arising from both intentional and unintentional NPS use.
Robin J Butterfield MBBS, MRCA ${ }^{1,2}$

Monica J Barratt $\mathrm{BSc}$ (Psych)(Hons), PhD ${ }^{3,4}$

Nadine Ezard MBBS, PhD, FAChAM ${ }^{1,2}$

Richard O Day MBBS (Hons), MD, FRACP ${ }^{1.2}$

1St Vincent's Hospital Sydney, NSW. 2 St. Vincent's Clinical School, University of New South Wales, Sydney, NSW. 3 National Drug and Alcohol Research Centre, University of New South Wales, Sydney, NSW.

4 National Drug Research Institute, Curtin University, Perth, WA.

robin.butterfield@svha. org.au

doi: 10.5694/mjal5.01058

\section{Drug-checking services}

Several European countries now provide drug analysis services, ${ }^{6}$ whereby individuals submit samples of their drugs to have their contents identified and analysed for purity. The results are provided to the consumer. The analytical facility can be based either on-site (eg, at large parties or festivals) or off-site. In some of the European services, brief health interventions aimed at reducing harm are offered to consumers simultaneously. Fast turn-around drug analysis services may have reduced harms resulting from recent episodes of mass intoxications at festival settings by:

- identifying the NPS and other contents of the pills or powders;

- monitoring NPS availability and use trends to enable an effective public health response;

- identifying emerging hazards from specific NPS and the formulations available;

- improving the knowledge base for effective clinical management of acute and chronic presentations;

- providing an opportunity for users to seek help, obtain health information to reduce potential harms and to offer options for individual behaviour change; and

- providing intelligence that could influence supply dynamics.

The optimal method for providing analytical services will depend largely on the social and legal context. In Austria and Switzerland, field workers from a non-government organisation conduct on-the-spot drug analysis to patrons at dance parties via a field laboratory equipped with high-pressure liquid chromatography machines. Samples are received directly from consumers and results are available to them within 20 minutes, accompanied by a brief intervention with referral if required. ${ }^{7}$ In the United Kingdom, amnesty bins are placed in party venues and a private, not-for-profit laboratory undertakes the analyses to add to a library of NPS. In larger nightclubs, on-site field workers use infra-red mass spectrometry to compare the drugs received with the database. Local accords between police, public health officials and the nightlife venue operators are required to ensure successful integrated services. A network of 26 drug-checking sites in the Netherlands is incorporated into the Ministry of Health as part of a national surveillance system. ${ }^{8}$ This service offers immediate results of quick office tests to potential users, with intervention and referral if necessary. Subsequently, 
samples are sent for more accurate spectrometric analysis, and results are available within a week. Most importantly, results are incorporated into the national surveillance system and are monitored for trends in emerging substances, and results are used to inform public policy and practice. While the direct prevention of deaths has not been documented, dangerous pills or powders identified by checking systems in the Netherlands have quickly disappeared from the Dutch markets following the launch of warning campaigns. ${ }^{9}$

Discovering new compounds that endanger life is unlikely to benefit the user after he or she has taken the drug except in ruling out other causes for developing severe syndromes. However, as more becomes known about the psychopharmacology of specific compounds, best-practice treatment algorithms can be created. If backed up by a sensible brief intervention, such a service might see users of drugs more engaged in caring for their health, recognising problem substance use, and seeking help. From a public health perspective, information on new compounds can be used to monitor emerging trends and inform prevention activities.

\section{Problems to overcome}

Several hurdles need to be overcome before drug checking can be established in Australia. Drug sellers may view the service as an opportunity to have their drugs checked before they distribute them. As in Europe, drug-checking services would need to ensure they are not complicit in aiding drug distribution. There may also be a misconception among users of the service that by having their drugs "checked", their use is condoned or seen as safe. Existing drug-checking services deal with this misconception through careful engagement with service users to explain that all drug use is risky and that the only completely safe option is to avoid drug use. This message is more likely to be heeded by users of drug-checking services who are receiving advice about harm reduction that is individually tailored to their personal characteristics and the known characteristics of the drugs they may consume.

As happens overseas, Australian drug-checking services will need to be provided in multiple sites in both metropolitan and regional locations. Transport problems would need to be solved to provide access to services outside metropolitan areas.

Effective laboratory testing is expensive; without adequate investment, the drug-checking service may be restricted to ad hoc sites or subject to unreliable testing techniques. Indeed, drug-checking interventions were conducted in Australia over a decade ago, ${ }^{10}$ but, at that time, on-site testing technology was restricted to colour reagent test kits, which are not reliable enough nor able to detect the larger number of substances currently available. In 2016, for an investment of under $\$ 200000$ (based on the costs of a high-performance liquid chromatography machine and employing a Scientist and Drug and Alcohol Counsellor), a mobile laboratory could be set up and attached to existing peer-run harmreduction services, supported by existing full laboratories providing in-kind support. While there are expenses associated with the ongoing running of such a service, the costs of trialling this kind of intervention are relatively low with the use of currently available technology that has been field-tested in other countries.

In addition, handling materials that are suspected of being illegal substances is prohibited by law; there are harm-reduction services currently operating in Australia that have been provided with an exemption for service staff and clients, such as Sydney's medically supervised injecting centre. Therefore, providing a drug-checking service would not require a radical shift in national drug policy, but would require cooperation between health and police stakeholders. radical shift in national drug policy, but would require cooperation between health and police stakeholders"

\section{Conclusions}

An important public health need in Australia could be met by providing an easily accessed drug-checking service that provides reliable and fast information to consumers about the content of drugs, along with non-judgemental harm-reduction advice. A further advantage is the ability of such a service to track the appearance of NPS on the market rapidly, ${ }^{8}$ which is helpful and sometimes necessary if we are to respond more effectively to NPS-related harm. Such a service could mitigate the severity and impact of situations that commonly overwhelm emergency departments around Australia by rapidly disseminating information about NPS to (a) potential consumers, warning them about specific products and batches, and (b) clinicians, guiding them on the predicted toxidrome and management of affected patients.

Concerns about the unintended consequences of providing a drug-checking service include legitimisation of the use of drugs, civil responsibility of the drug checkers towards consumers of tested drugs, and the use of the service by drug sellers as a quality control mechanism. Such concerns have not been supported by evidence in the European context. ${ }^{11}$ In Europe, the service is used in tandem with opportunistic brief interventions that provide a moment for education on health and harm reduction, and reduces the delay to treatment for problem drug use. Further experience with services of this kind is required to ascertain the feasibility, acceptability and effectiveness in inducing behaviour change of various models of drug checking in the Australian context, which is different from the Dutch context (eg, there is no threat of prosecution for using these drugs in the Netherlands). Therefore, it is very important that a high-quality research trial of drug checking in Australia be conducted.

Acknowledgements: Monica Barratt is the recipient of a National Health and Medical Research Council Early Career Researcher Fellowship (APP1070140).

Competing interests: No relevant disclosures.

(c) 2016 AMPCo Pty Ltd. Produced with Elsevier B.V. All rights reserved.

References are available online at www.mja.com.au. 
1 McCutcheon DS, Oosthuizen FJ, Hoggett KA, Fatovich DM. A bolt out of the blue: the night of the blue pills. Med J Aust 2015; 202: 543. https://www.mja.com.au/journal/2015/202/ 10/bolt-out-blue-night-blue-pills

2 Duffy C. Stereosonic death: emergency doctor warns worst is yet to come following music festival drug overdose [television program]. 730. Australian Broadcasting Commission; 2015: 30 Nov. http://www.abc.net.au/7.30/content/2015/s4363001.htm (accessed Dec 2015).

3 European Monitoring Centre for Drugs and Drug Addiction. European drug report 2015: trends and developments. Lisbon: EMCDDA, 2015. http://www.emcdda.europa.eu/edr2015 (accessed Dec 2015).

4 Burns L, Roxburgh A, Bruno R, Van Buskirk J. Monitoring drug markets in the internet age and the evolution of drug monitoring systems in Australia. Drug Test Anal 2014; 6: 840-845.

5 Irvine RJ, Kostakis C, Felgate PD, et al. Population drug use in Australia: a wastewater analysis. Forensic Sci Int 2011; 210: 69-73.

6 Trans European Drug Information (TEDI). Guidelines for drug checking methodology. Barcelona: TEDI, 2012. http://newip.
safernightlife.org/pdfs/digital_library/Guidelines\%20for\% 20Drug\%20Checking\%20Methodology.pdf (accessed Dec 2015).

7 Hungerbuehler I, Buecheli A, Schaub M. Drug checking: a prevention measure for a heterogeneous group with high consumption frequency and polydrug use - evaluation of Zurich's drug checking services. Harm Reduct J 2011; 8: 16.

8 Brunt TM, Niesink RJ. The Drug Information and Monitoring System (DIMS) in the Netherlands: implementation, results, and international comparison. Drug Test Anal 2011; 3: 621-634.

9 van Laar M, Cruts C, van Gageldonk A, et al. The Netherlands drug situation 2006. Report to the EMCDDA by the Reitox National Focal Point. Utrecht: Trimbos Institute, 2006. http://www.emcdda.europa.eu/attachements.cfm/att_44964_ EN_NR2006nl.pdf (accessed Jan 2016).

10 Camilleri AM, Caldicott D. Underground pill testing, down under. Forensic Sci Int 2005; 151: 53-58.

11 Benschop A, Rabes M, Korf DJ. Pill testing, ecstasy and prevention: a scientific evaluation in three European cities. Amsterdam: Rozenberg Publishers, 2002. 